\title{
Educational delay and attainment in persons with neurofibromatosis 1 in Denmark
}

\author{
Karoline Doser $^{1} \cdot$ Line Kenborg $^{2}$ • Elisabeth Wreford Andersen ${ }^{1} \cdot$ Pernille Envold Bidstrup ${ }^{1} \cdot$ Anja Kroyer $^{2}$. \\ Hanne Hove $^{3}$ • John Østergaard ${ }^{4}$ • Sven Asger Sørensen ${ }^{5}$. Christoffer Johansen ${ }^{1,6}$ • John Mulvihill ${ }^{7}$. \\ Jeanette Falck Winther ${ }^{2,8} \cdot$ Susanne Oksbjerg Dalton ${ }^{1}$
}

Received: 24 May 2018 / Revised: 20 December 2018 / Accepted: 24 January 2019 / Published online: 28 February 2019

(c) European Society of Human Genetics 2019

\begin{abstract}
Most research on psychosocial consequences of neurofibromatosis type 1 (NF1) has focused on the relationship between disease factors and cognitive functioning. NF1 may impair domains of learning and attention, resulting in low academic performance. This study is the first nationwide population-based cohort study to investigate educational attainment and delay in completing mandatory school by persons with NF1. Educational information was collected from 550 persons at the age of 30 (born 1965-1984). They were diagnosed with NF1 in Denmark and compared to a cohort of NF1-free persons matched on gender and age $(n=4295)$. Multinomial logistic models were applied to estimate odds ratios (ORs) for obtaining short ( $\leq 9$ years) or medium (10-12 years) education compared to long education ( $>12$ years) by the age of 30 years. We calculated the probability of graduating $9^{\text {th }}$ year of mandatory school at different ages in 932 persons with NF1 and 7962 NF1-free persons (born 1965-2000) using quantile regression. The OR of educational completion for short- and medium-term education was three fold (95\% CI 2.55-3.99) and 1.29 fold (95\% CI 0.99-1.69) higher, respectively, for persons with NF1 than NF1-free persons after adjusting for birth year, gender, psychiatric and somatic morbidity and mother's education. Persons with NF1 were significantly delayed in graduating mandatory school education compared to NF1-free persons. When $90 \%$ of persons have graduated, persons with NF1 were 1.2 times older than the NF1-free persons. Experiencing delays in mandatory school likely affect further educational achievements and may impair employment and entering work force.
\end{abstract}

\section{Introduction}

Neurofibromatosis 1 (NF1) is one of the most common Mendelian diseases with a worldwide incidence of 1 per

These authors contributed equally: Jeanette Falck Winther, Susanne Oksbjerg Dalton

Karoline Doser

kdoser@cancer.dk

1 Survivorship Unit, Danish Cancer Society Research Center, Copenhagen, Denmark

2 Childhood Cancer Research Group, Danish Cancer Society Research Center, Copenhagen, Denmark

3 The RAREDIS Database, Centre for Rare Diseases, Copenhagen University Hospital and Aarhus University, Aarhus Denmark
2500 to 3000 persons [1]. This autosomal dominant disorder is caused by a germline, inactivating variant in the neurofibromin gene located at chromosome 17q11.2. NF1 is associated with a variety of clinical manifestations and complications leading to a reduced life expectancy [2-4]. Cardinal signs include café-au-lait macules, neurofibromas, axillary and inguinal freckling, optic glioma, iris lisch nodules and dysplasia of the long bone or sphenoid [5]. Further, neurologic complications are prevalent [6].

4 Center for Rare Disease, Aarhus University Hospital, Aarhus, Denmark

5 Copenhagen University, Copenhagen, Denmark

6 Oncology Clinic, Finsen Center, Rigshospitalet, 5073 Copenhagen, Denmark

7 University of Oklahoma Health Sciences Center, Oklahoma City, OK, USA

8 Department of Clinical Medicine, Faculty of Health, Aarhus University, Aarhus, Denmark 
Although NF1 is believed to be completely penetrant by age 20 , pleiotrophy and expressivity is extremely variable, even among family members with the same genetic variation [7] and it is not possible to predict which symptoms will develop in any affected individual [8].

Neurofibromin (the altered protein in NF1) plays a role in brain function [9] and cognitive impairment is considered the most common neurologic feature of NF1 in childhood [6]. Of 26 adolescents with NF1, 19\% had intellectual functioning below average, although the degree of impairment was mild [10]. Furthermore, in 86 children with NF1, 75\% performed significantly lower than grade peers in at least one of the domains of spelling, mathematics, technical reading or comprehensive reading, indicating learning disabilities [11], while $63 \%$ of another 81 NF1 children had attention difficulties and $38 \%$ fulfilled the diagnostic criteria for attention deficit hyperactivity disorder (ADHD) [12]. Compared with age- and gender-matched NF1-free controls, 20 young adults with NF1 showed significantly more problems with visualspatial skills, auditory long-term memory, and language [13]. In a recent study, several prevalent somatic diseases, for example cancer and bone disease, as well as psychiatric conditions, such as ADHD and autism were described in the population of Danes with NF1 [14] and it may be expected that the presence of somatic, psychiatric but especially cognitive conditions will interfere with academic achievement and ultimately, educational attainment. However, few studies have examined educational achievement of persons with NF1 [11, 15-20], addressing mostly academic difficulties and the specific disorders that may challenge children with NF1 in school such as visual-spatial deficits, dyslexia and dyscalculia, attention deficits, and executive dysfunction. Academic performance (including reading, spelling, math reasoning, and listening comprehension) was significantly poorer in a hospital sample of 79 children with NF1 when compared to their 46 unaffected siblings [20]. However, beside the overall academic attainment, the trajectory of education also plays an important role. Higher rates of repeating first grade and poorer academic prognosis was observed in 116 children with NF1 compared to 80 normally developed children [21]. Semi-structured interviews revealed that $33 \%$ of 60 adults attained less than high school graduation [19], a rate similar among 228 adults with NF1 for completing under 10 years of education [22]. In Denmark graduation from year 9 represents the endpoint of lower secondary school and indicates a turning point for potentially further education and career building. Academic performance and the timely achievement of educational milestones are important for social and economic independence as well as participation in society later in life [21].
However, no large quantitative population-based study has investigated the completed educational level among adults with NF1 and no information about delays in achieving academic levels is available. Former research has been mainly cross-sectional based on self-reports or family report [18], often without a comparison group. Further details on overall educational achievement should improve support for persons with NF1 and their families.

To our knowledge, this is the first nationwide population-based cohort study of persons with NF1 and a population-based comparison cohort using the nationwide registers in Denmark that provide virtually complete follow-up. The study aims to address the two research questions: Which level of education do persons with NF1 achieve in Denmark and do they attain less education than NF1-free persons? In addition, do children with NF1 graduate from lower secondary school, which is required to initiate youth education (and subsequently any further education) at a later age than NF1-free children? We hypothesized that any association was modified by medical and psychiatric morbidity, which along with birth year, gender and mother's education may modify the association.

\section{Materials and methods}

\section{Study populations}

We conducted a nationwide registry-based cohort study. In Denmark, all residents have been recorded in the Central Population Register (CPR) since 1 April 1968 [23] with unique personal identification numbers that ensure accurate linkage of information among all national registries.

The population register-based NF1 cohort consisted of all persons hospitalized for or treated with existing neurofibromatosis as registered in the National Hospital Register [24] from 1977 and onwards, as well as all patients affiliated to the two national Centers of Rare Disease situated at the National State Hospital Rigshospitalet in Copenhagen and at the University Hospital in Aarhus, Denmark. Overall, 2517 persons with NF1 were identified and registered due to diagnosis of or treatment for NF1, 2421 from the National Hospital Register and 637 from the Centers of Rare Disease (541 were in both sources).

A population-based NF1-free comparison cohort was randomly selected in the CPR based on gender and month and year of birth of the persons with NF1, in an approximate 10 to 1 ratio. From the CPR, we identified parents of the persons with NF1 and those in the comparison cohort, which numbers 25,128 persons. We extracted information on gender and date of birth, date of death or emigration for both study cohorts and the parents. 


\section{Information on education and medical and psychiatric disease}

\section{Educational attainment}

We obtained the highest level of completed education from the Danish Education Registry, which includes information on all educational programs in the country since 1981 and ascertains the highest level of completed education on a yearly basis [25]. Thus, the study period with available data is 1981-2015. The Danish educational system currently consists of 9 years mandatory basic school (primary and lower secondary levels of education for children aged 6-16), youth education (high school/ upper secondary education qualifying for higher education), vocational/technical training which qualifies for vocational positions, and higher education (consisting of $2-3 \frac{1}{2}$ year bachelor and professional programs and 5-6 years graduate university program of master and $\mathrm{PhD}$ level) [26]. We grouped education into the following categories, with the corresponding International Standard Classification of Education (ISCED-2011) codes: short (ISCED codes $1-2, \leq 9$ years), medium (ISCED 3-4, 1012 years), and long (ISCED 5-8,> 12 years) [27]. For persons born before 1958, mandatory basic school was 7 years, which will apply for some parents. To investigate the highest educational attainment, a sub-sample was restricted to persons born 1965 to 1984 (NF1 cohort, $N=$ 550; NF1-free cohort, $N=4295$ ), in order to obtain information among persons aged 30 or older when most persons have completed their education. Since the education level was missing for a considerable proportion of the population born before 1920, we only included persons born after 1965 to reduce missing information on parental education information. This sub-analysis on age at completion of the mandatory $9^{\text {th }}$ grade, was restricted to those persons with NF1 who completed the $9^{\text {th }}$ grade of mandatory school. As most Danish children are expected to finish $9^{\text {th }}$ grade in the year they turn 16 , persons born 1965-2000 were included (NF1 cohort, $N=932$; NF1free cohort, $N=7962$ ). We obtained the date at completion of the mandatory $9^{\text {th }}$ grade for both the NF1 cohort and the NF1-free comparison cohort and computed the exact age at graduation (Fig. 1).

\section{Somatic diseases}

From the Danish National Patient Registry [24], we obtained information on all hospital contacts for cancer, bone diseases, diseases of the nervous system, diseases of the circulatory system and other somatic disorders (for ICD-8 and ICD-10 codes, see Table 1), which are the most prevalent severe conditions in Danish persons with NF1 [14].

\section{Psychiatric diagnoses}

Information regarding hospital contacts with psychiatric diagnoses was retrieved from the Danish Psychiatry Research Register [28] from 1969 onwards supplemented with information from the National Patient Registry. We grouped the psychiatric diagnoses into anxiety, depression, ADHD, autism and other psychiatric disease (ICD-10 codes are included in Table 1 and Table 3).

\section{Statistical analyses}

Descriptive characteristics of the persons with NF1 as well as the population-based matched comparison subjects and differences in distributions were evaluated by Chi-square tests for both educational attainment at age 30 or more and delay in finalizing $9^{\text {th }}$ grade of mandatory school.

\section{Educational attainment}

Multinomial logistic regression models were used to investigate the association between NF1 status and education using long education as the reference category for short or medium education and presenting the results as conditional odds ratio (COR). To account for some persons with NF1 being related, we applied robust variance estimation adjusting for within cluster correlations (the clusters being families) [29].

Three multinomial logistic regression models were developed stepwise adding the potential confounders and effect modifiers. Model 1 included the exposure NF1 status and birth year (in 3-4 year blocks from 1965-1984) and gender, and Model 2 further included the educational level of the mother. Since more fathers than mothers had missing education we only included mothers' education as confounder in the analyses. Finally, in Model 3, we further added information about medical and psychiatric disease, which may be considered as effect modificator [14, 30, 31] of the NF1 effect on the attained education level. Possible effect modification was examined in Model 3 by adding the interactions between NF1 status and birth year, medical disease, psychiatric diagnosis, mother's education and gender one at a time.

In order to display the effect of NF1 on the probability of educational attainment, we calculated the differences between the probability of education for persons with NF1 and NF1free persons for each birth period based on Model 3.

\section{Delay in graduation from lower secondary school}

Non-parametric estimates of the cumulative probability functions by NF1-status were calculated to assess graduating from 9th grade at different ages (estimates by 1-Kaplan Meier). Quantile regression was used to model quantiles of 


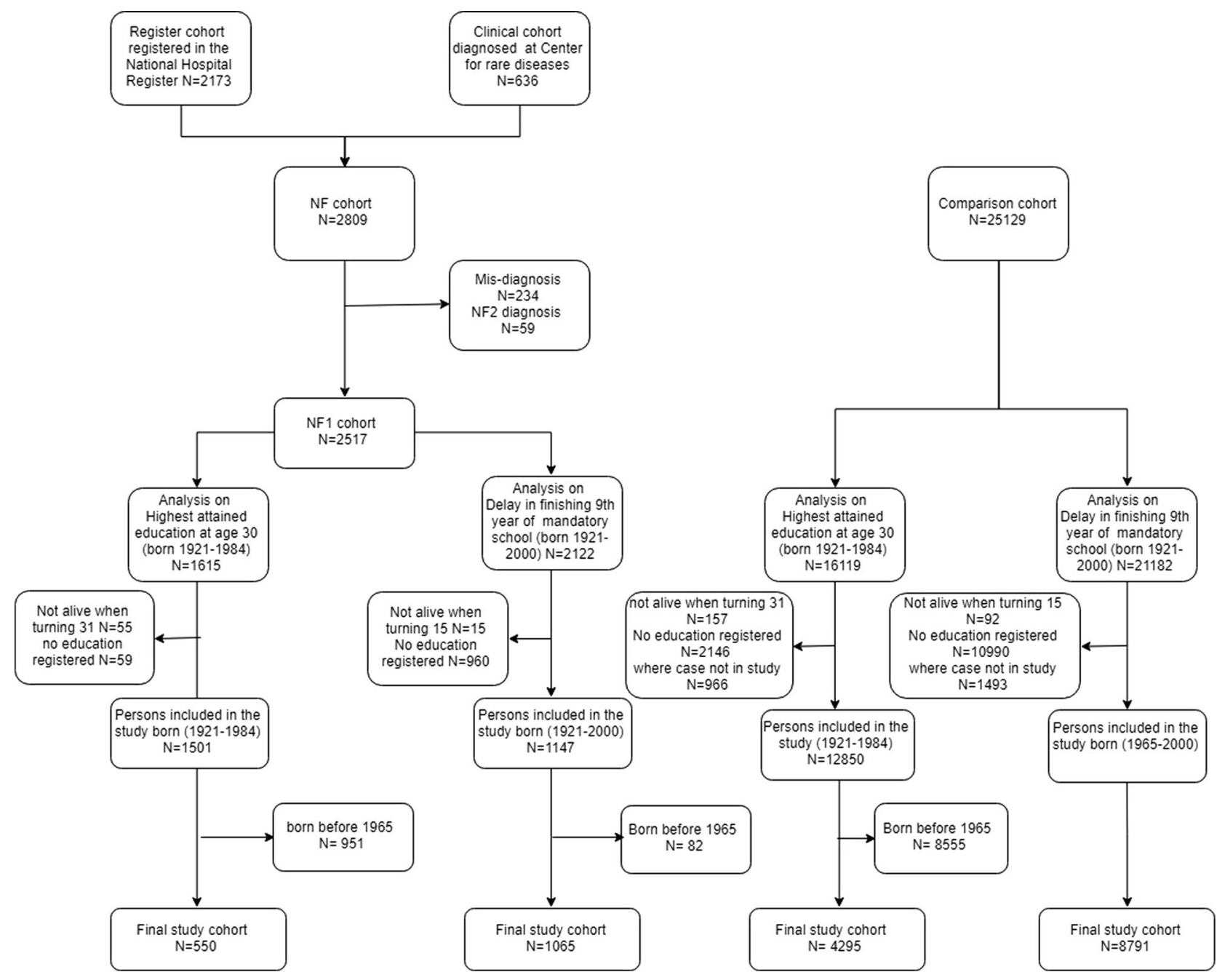

Fig. 1 Cohort description

the age at graduation adjusting for possible confounders. The analyses included the $10^{\text {th }}, 25^{\text {th }}, 50^{\text {th }}, 75^{\text {th }}$ and $90^{\text {th }}$ percentiles. Three different regression models were fitted: Model 1 included the exposure NF1 and the potential confounders birth year (in 5-year blocks between 1965 and 2002) and gender, Model 2 additionally included mother's education. Finally, Model 3 added information about medical and psychiatric disease to investigate possible effect modification.

\section{Sensitivity analyses}

Missing information on maternal education was about $9 \%$. Sensitivity analyses were performed using multiple imputations, a simulation-based technique to treat missing data was run on 50 imputed data sets. Multinomial logistic analysis were carried out in STATA version 14 [32]. The quantile regression analysis were carried out in SAS software [33]. The study was approved by the Danish Protection Agency (Record 2014-41-2935) and the Human
Research Protection Office (HRPO) (A-18370.i) (June 2015/2016).

\section{Results}

\section{Highest attained education}

The NF1 cohort consisted of 550 adult persons who were matched to 4295 NF1-free persons with a proportion of 56 and $57 \%$ females, respectively. Overall, more persons with NF1 had short education and fewer a long education compared to the NF1-free cohort (41\% versus 20 and $21 \%$ versus $34 \%$, respectively; $p<0.0001)$. Additional characteristics of the cohorts are shown in Table 1.

The adjusted odds ratio of short education was three-fold increased for persons with NF1 (COR 3.00, 95\% CI: 2.553.99, Model 3), while COR of medium education was 1.29 for the persons with NF1 (95\% CI 0.99-1.69, Model 3) compared to NF1-free persons (Table 2). In general, the 


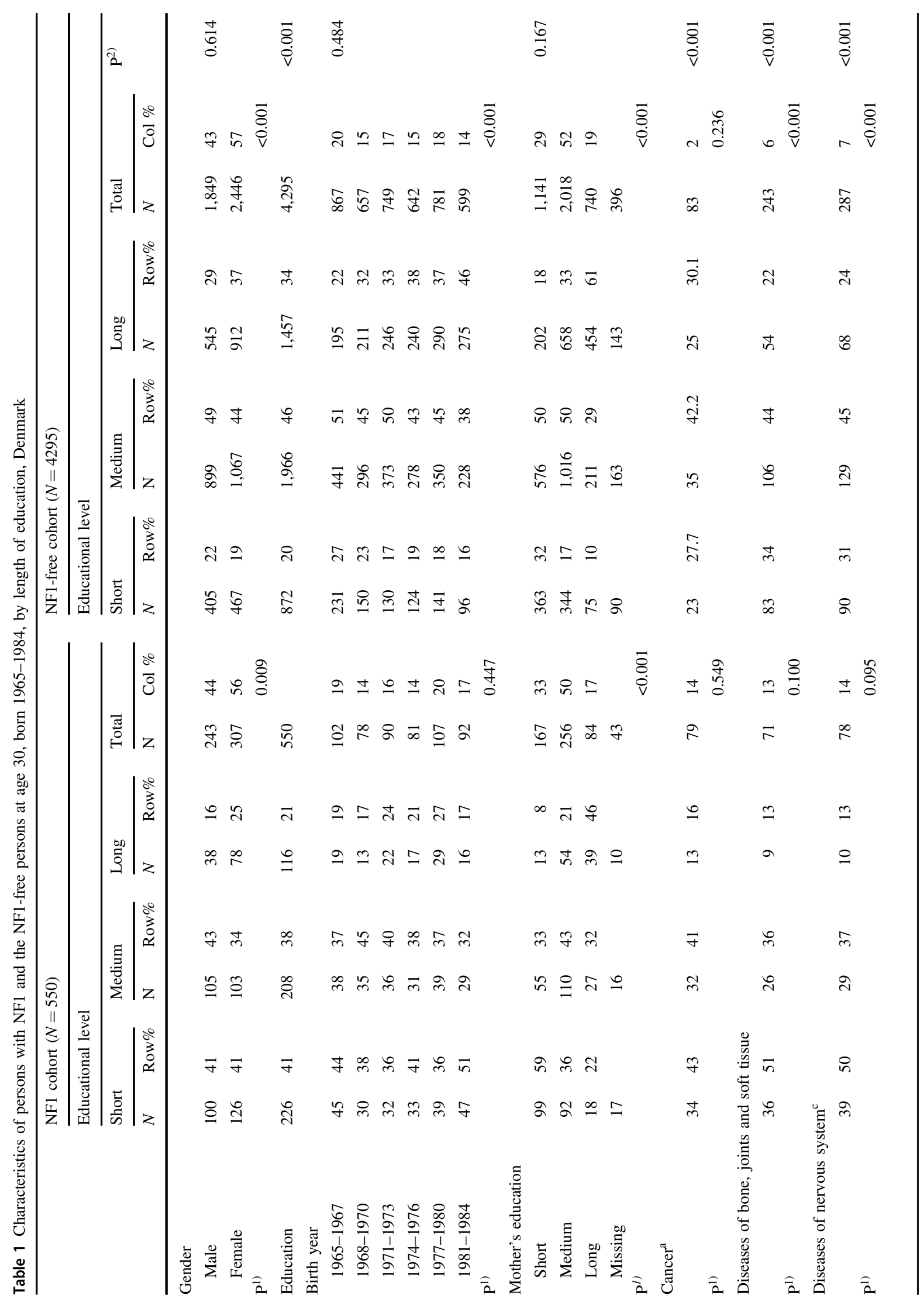




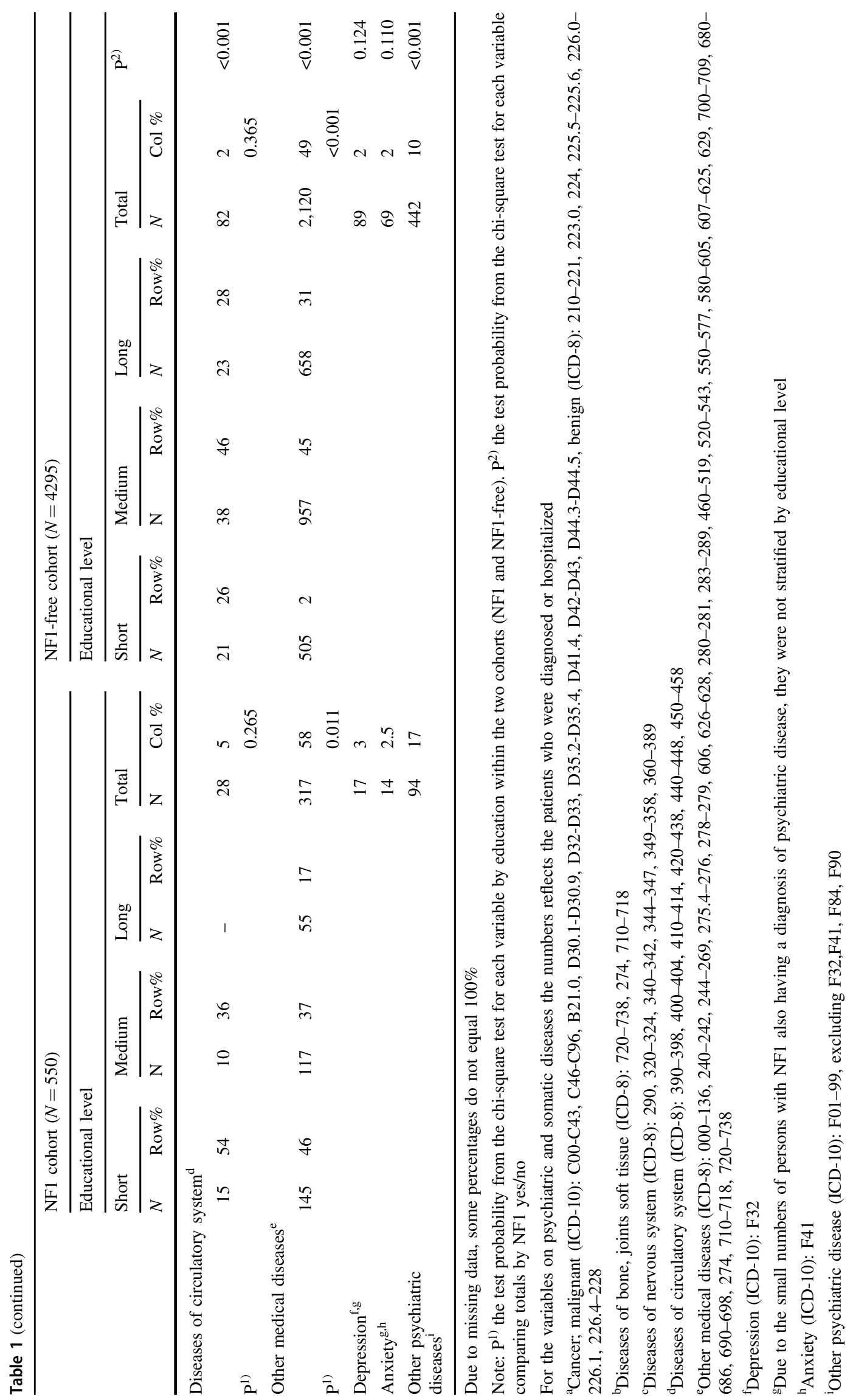


Table 2 Multivariate odds ratios (OR) with 95\% confidence intervals (CI) for education levels among persons with NF1 born 1965-1984, compared to NF1-free persons using long education as the reference level, Denmark, 1981-2015

\begin{tabular}{|c|c|c|c|c|}
\hline & \multicolumn{2}{|c|}{ Short education } & \multicolumn{2}{|c|}{ Medium education } \\
\hline & OR & CI & OR & $\mathrm{CI}$ \\
\hline \multicolumn{5}{|l|}{ NF1 } \\
\hline No & 1.00 & Reference & 1.00 & Reference \\
\hline Yes & 3.00 & $2.25-3.99$ & 1.29 & $0.99-1.69$ \\
\hline \multicolumn{5}{|l|}{ Gender } \\
\hline Male & 1.00 & Reference & 1.00 & Reference \\
\hline Female & 0.58 & $0.48-0.70$ & 0.60 & $0.52-0.69$ \\
\hline \multicolumn{5}{|l|}{ Birth year } \\
\hline 1965-1967 & 1.00 & Reference & 1.00 & Reference \\
\hline 1968-1970 & 0.60 & $0.44-0.81$ & 0.72 & $0.56-0.93$ \\
\hline 1971-1973 & 0.48 & $0.36-0.66$ & 0.75 & $0.59-0.96$ \\
\hline 1974-1976 & 0.46 & $0.33-0.63$ & 0.61 & $0.47-0.79$ \\
\hline 1977-1980 & 0.37 & $0.27-0.50$ & 0.59 & $0.46-0.76$ \\
\hline 1981-1984 & 0.30 & $0.22-0.41$ & 0.44 & $0.34-0.57$ \\
\hline \multicolumn{5}{|c|}{ Mother's education } \\
\hline Short & 1.00 & Reference & 1.00 & Reference \\
\hline Medium & 0.30 & $0.24-0.37$ & 0.56 & $0.47-0.68$ \\
\hline Long & 0.10 & $0.07-0.13$ & 0.18 & $0.14-0.22$ \\
\hline \multicolumn{5}{|l|}{ Cancer } \\
\hline No & 1.00 & Reference & 1.00 & Reference \\
\hline Yes & 1.28 & $0.78-2.07$ & 1.14 & $0.74-1.77$ \\
\hline \multicolumn{5}{|c|}{ Diseases of bone, joints and soft tissue } \\
\hline No & 1.00 & Reference & 1.00 & Reference \\
\hline Yes & 2.21 & $1.56-3.13$ & 1.46 & $1.05-2.02$ \\
\hline \multicolumn{5}{|c|}{ Diseases of nervous system } \\
\hline No & 1.00 & Reference & 1.00 & Reference \\
\hline Yes & 1.83 & $1.32-2.55$ & 1.43 & $1.06-1.91$ \\
\hline \multicolumn{5}{|c|}{ Diseases of circulatory system } \\
\hline No & 1.00 & Reference & 1.00 & Reference \\
\hline Yes & 1.49 & $0.80-2.79$ & 1.30 & $0.76-2.23$ \\
\hline \multicolumn{5}{|c|}{ Other medical diseases } \\
\hline No & 1.00 & Reference & 1.00 & Reference \\
\hline Yes & 1.54 & $1.28-1.85$ & 1.15 & $0.99-1.33$ \\
\hline \multicolumn{5}{|l|}{ Depression } \\
\hline No & 1.00 & Reference & 1.00 & Reference \\
\hline Yes & 1.72 & $0.89-3.35$ & 1.14 & $0.60-2.16$ \\
\hline \multicolumn{5}{|l|}{ Anxiety } \\
\hline No & 1.00 & Reference & 1.00 & Reference \\
\hline Yes & 2.06 & $0.94-4.53$ & 1.77 & Reference \\
\hline \multicolumn{5}{|c|}{ Other psychiatric diseases } \\
\hline No & 1.00 & Reference & 1.00 & Reference \\
\hline Yes & 4.37 & $3.24-5.88$ & 1.41 & $1.06-1.88$ \\
\hline Intercept & 2.69 & $2.05-3.54$ & 4.77 & $3.75-6.07$ \\
\hline
\end{tabular}

$O R$ odds ratio, $C I$ confidence intervals

The estimated standard errors used to calculate $95 \%$ confidence intervals are robust taking into account that some of the subjects are related

Model is adjusted for gender, birth year, mother's length of education, medical and psychiatric diseases

adjustments from model 1 to 3 only resulted in minor changes to the COR estimates and no effect modification of NF1 on education according to gender, birth year, medical

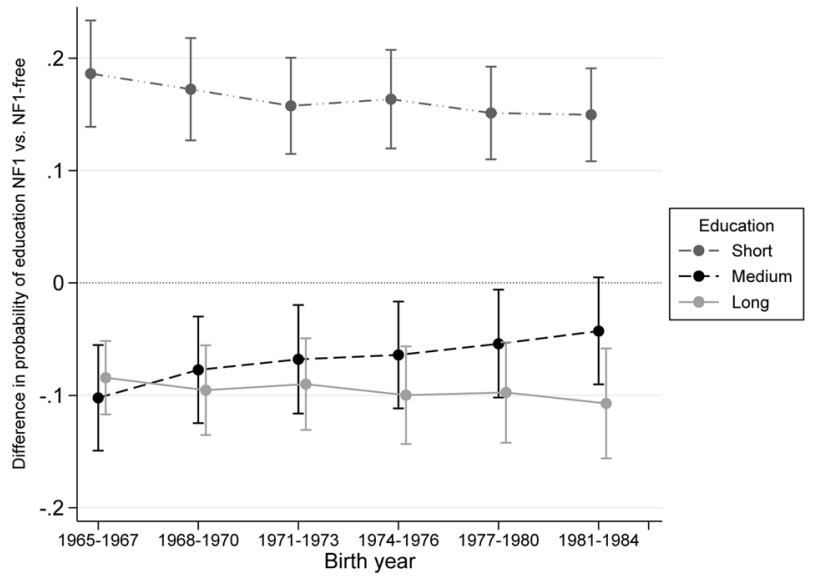

Fig. 2 Effect of NF1 status on the probability of length of education by birth year (1965-1984) among 550 adults with NF1 and 4295 NF1free persons in Denmark, 1981-2015

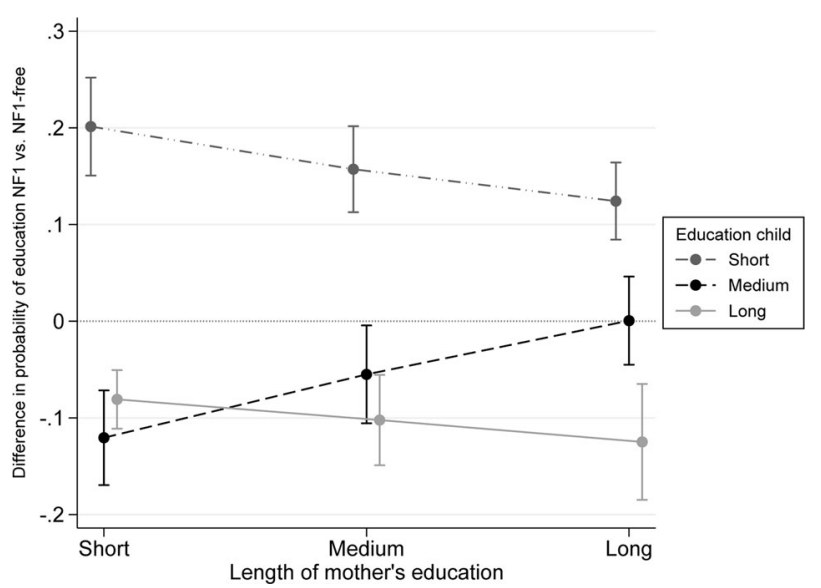

Fig. 3 Effect of NF1 on the probability of education by mother's length of education (in birth year 1974-1976) among 550 adults with NF1 and 4295 NF1-free persons in Denmark, 1981-2015

disease, depression, anxiety, or other psychiatric disorders was observed (data not shown).

Persons with NF1 had a significant and approximate 0.20 higher probability of having attained short education and approximately 0.15 lower probability of long education compared to the NF1-free population (Fig. 2). For the birth cohort born in 1974-76, mother's education was associated with attained education in both groups, but having NF1 increased the probability of having short education at age 30 significantly irrespective of mother's education (Fig. 3).

Only minimal differences from the main results were seen in the sensitivity analyses by using multiple imputations albeit with narrower CIs (results not shown).

\section{Delays in graduation from lower secondary school}

In total, 932 persons with NF1 and 7962 NF1-free persons born 1965-2000 graduated from $9^{\text {th }}$ grade between 
Table 3 Descriptive characteristics of the 1065 Danish persons with NF1 and the 8791 NF1-free persons, born 1965-2000, graduated from grade 9 in Denmark 1981-2015

\begin{tabular}{|c|c|c|c|c|c|c|c|c|}
\hline & \multicolumn{4}{|c|}{ NF1 cohort } & \multicolumn{4}{|c|}{ NF1-free cohort } \\
\hline & $N$ & $\%$ & Grad. & Person-years & $N$ & $\%$ & Grad. & Person-years \\
\hline Total & 1,065 & & 932 & 2,734 & 8,791 & & 7,962 & 19,274 \\
\hline \multicolumn{9}{|l|}{ Gender } \\
\hline Male & 529 & 50 & 454 & 1,416 & 4,426 & 50 & 3,897 & 10,216 \\
\hline Female & 536 & 50 & 478 & 1,318 & 4,365 & 50 & 4,065 & 9,058 \\
\hline \multicolumn{9}{|c|}{ Pearson $\chi^{2}(1)=0.17$ Pr. $=0.68$} \\
\hline \multicolumn{9}{|l|}{ Birth year } \\
\hline $1965-1969$ & 107 & 10 & 102 & 260 & 960 & 11 & 916 & 2,187 \\
\hline 1970-1974 & 151 & 14 & 144 & 341 & 1,248 & 14 & 1,208 & 2,687 \\
\hline 1975-1979 & 136 & 13 & 128 & 351 & 1,025 & 12 & 996 & 2,278 \\
\hline 1980-1984 & 133 & 13 & 119 & 412 & 907 & 10 & 869 & 2,082 \\
\hline 1985-1989 & 128 & 12 & 115 & 384 & 934 & 10 & 890 & 2,249 \\
\hline 1990-1994 & 164 & 15 & 153 & 465 & 1,375 & 16 & 1,314 & 3,330 \\
\hline $1995-2002$ & 246 & 23 & 171 & 520 & 2,342 & 27 & 1,769 & 4,461 \\
\hline \multicolumn{9}{|c|}{ Pearson $\chi^{2}(6)=12.20 \operatorname{Pr}=0.058$} \\
\hline \multicolumn{9}{|c|}{ Mother's education } \\
\hline Short & 343 & 32 & 298 & 891 & 2,234 & 25 & 2,003 & 5,381 \\
\hline Medium & 502 & 47 & 439 & 1,317 & 4,273 & 49 & 3,914 & 9,199 \\
\hline Long & 189 & 18 & 168 & 434 & 2,097 & 24 & 1,896 & 4,211 \\
\hline Missing & 31 & 3 & & & 187 & 2 & & \\
\hline \multicolumn{9}{|c|}{ Pearson $\chi^{2}(3)=35.11 \operatorname{Pr}=0.000$} \\
\hline \multicolumn{9}{|c|}{ Cancer $^{\mathrm{a}}$} \\
\hline Yes & 57 & 5 & 50 & 148 & 49 & 1 & 48 & 102 \\
\hline \multicolumn{9}{|c|}{ Pearson $\chi^{2}(1)=205.26 \operatorname{Pr}=0.000$} \\
\hline \multicolumn{9}{|c|}{ Diseases of bone, joints and soft tissue ${ }^{b}$} \\
\hline & 76 & 7 & 70 & 192 & 137 & 2 & 126 & 306 \\
\hline \multicolumn{9}{|c|}{ Pearson $\chi^{2}(1)=139.77 \operatorname{Pr}=0.000$} \\
\hline \multicolumn{9}{|c|}{ Diseases of nervous system ${ }^{c}$} \\
\hline & 155 & 15 & 126 & 479 & 468 & 5 & 406 & 1,136 \\
\hline \multicolumn{9}{|c|}{ Pearson $\chi^{2}(1)=136.68 \operatorname{Pr}=0.000$} \\
\hline \multicolumn{9}{|c|}{ Diseases of circulatory system ${ }^{\mathrm{d}}$} \\
\hline & 25 & 2 & 19 & 62 & 34 & 0 & 32 & 72 \\
\hline \multicolumn{9}{|c|}{ Pearson $\chi^{2}(1)=61.37 \operatorname{Pr}=0.000$} \\
\hline \multicolumn{9}{|c|}{ Other medical diseases ${ }^{\mathrm{e}}$} \\
\hline & 533 & 50 & 461 & 1,442 & 3,071 & 35 & 2,746 & 6,938 \\
\hline \multicolumn{9}{|c|}{ Pearson $\chi^{2}(1)=93.54 \operatorname{Pr}=0.000$} \\
\hline \multicolumn{9}{|c|}{ Anxiety ${ }^{\mathrm{f}}$} \\
\hline & 7 & 1 & 5 & 17 & 48 & 1 & 42 & 123 \\
\hline Pearson $\chi^{2}(1)$ & $=0.645$ & & & & & & & \\
\hline Autism ${ }^{\mathrm{g}}$ & & & & & & & & \\
\hline & 23 & 2 & 15 & 92 & 92 & 1 & 75 & 295 \\
\hline Pearson $\chi^{2}(1)$ & $r=0.00$ & & & & & & & \\
\hline $\mathrm{ADHD}^{\mathrm{h}}$ & & & & & & & & \\
\hline & 50 & 5 & 39 & 114 & 114 & 1 & 94 & 324 \\
\hline Pearson $\chi^{2}(1)$ & $r=0.00$ & & & & & & & \\
\hline Autism/ADHD & & & & & & & & \\
\hline & 69 & 7 & 50 & 198 & 199 & 2 & 163 & 601 \\
\hline Pearson $\chi^{2}(1)$ & $r=0.00$ & & & & & & & \\
\hline Depression an & chiatric & & & & & & & \\
\hline & 172 & 16 & 128 & 499 & 497 & 6 & 405 & 1,432 \\
\hline Pearson $\chi^{2}(1)$ & $\operatorname{Pr}=0.0$ & & & & & & & \\
\hline
\end{tabular}


Table 3 (continued)

\begin{tabular}{|c|c|c|c|c|c|c|c|c|}
\hline & \multicolumn{4}{|c|}{ NF1 cohort } & \multicolumn{4}{|c|}{ NF1-free cohort } \\
\hline & $N$ & $\%$ & Grad. & Person-years & $N$ & $\%$ & Grad. & Person-years \\
\hline \multicolumn{9}{|c|}{ Age at graduation (years) } \\
\hline $14-15$ & & & 10 & 1,047 & & & 46 & 8,622 \\
\hline $15-15.5$ & & & 44 & 501 & & & 579 & 4,090 \\
\hline $15.5-16$ & & & 278 & 413 & & & 3,189 & 3,074 \\
\hline $16-16.5$ & & & 348 & 235 & & & 3,250 & 1,260 \\
\hline $16.5-17$ & & & 170 & 110 & & & 703 & 345 \\
\hline $17-18$ & & & 77 & 83 & & & 186 & 315 \\
\hline $18-20$ & & & 5 & 346 & & & 9 & 1,568 \\
\hline
\end{tabular}

a Cancer; malignant (ICD-10): C00-C43, C46-C96, B21.0, D30.1-D30.9, D32-D33, D35.2-D35.4, D41.4, D42-D43, D44.3-D44.5, benign (ICD-8): 210-221, 223.0, 224, 225.5-225.6, 226.0-226.1, 226.4-228

${ }^{\mathrm{b}}$ Diseases of bone, joints soft tissue (ICD-8): 720-738, 274, 710-718

${ }^{\mathrm{c}}$ Diseases of nervous system (ICD-8): 290, 320-324, 340-342, 344-347, 349-358, 360-389

${ }^{\mathrm{d}}$ Diseases of circulatory system (ICD-8): 390-398, 400-404, 410-414, 420-438, 440-448, 450-458

${ }^{\mathrm{e}}$ Other medical diseases (ICD-8): 000-136, 240-242, 244-269, 275.4-276, 278-279, 606, 626-628, 280-281, 283-289, 460-519, 520-543, 550$577,580-605,607-625,629,700-709,680-686,690-698,274,710-718,720-738$

${ }^{\mathrm{f}}$ Anxiety (ICD-10): F41, Depression (ICD-10): F32

${ }^{\mathrm{g}}$ Autism (ICD-10): F84

${ }^{\mathrm{h}} \mathrm{ADHD}$ (ICD-10): F90

${ }^{\mathrm{i}}$ Depression and other psychiatric disease (ICD-10): F01-99, excluding F41, F84, F90

1981 and 2014. As displayed in Table 3, a higher prevalence of psychiatric and somatic diagnoses were seen in persons with NF1 compared to that in NF1-free persons.

The cumulative probability of graduation from $9^{\text {th }}$ grade by NF1 status (Fig. 4a) showed that persons with NF1 finished their mandatory school education later than NF1free persons from around age of 15.5 years with an increasing age difference from then onwards. This was more pronounced for males than for females.

The effect of NF1 status on age at graduation from 9th grade increased with each percentile. After $10 \%$ of the persons with NF1 had graduated from $9^{\text {th }}$ grade, $5 \%(95 \%$ CI, 2-8\%) were older than the NF1-free persons adjusted for sex and birth year (Table 4). When half of the persons had graduated, $9 \%(95 \% \mathrm{CI}: 7-11 \%)$ were older than the NF1-free persons and when nearly all $(90 \%)$ had graduated $26 \%$ (95\% CI 19-33\%) of the persons with NF1 were older. Adjusting for mother's length of education marginally changed the estimate for the $90 \%$ quantile, whereas only adjusting for somatic and psychiatric disease did not change estimates (Table 4).

Looking specifically at the birth cohort 1975-79 at age 16 years, the probability of graduating from $9^{\text {th }}$ grade was 0.35 among boys with NF1 and 0.41 among girls with NF1, whereas it was 0.44 in boys and 0.55 in NF1-free girls, respectively (Fig. 4b).

\section{Discussion}

In this nationwide registry-based study, we found that persons with NF1 had a higher likelihood of attaining only short and medium education and that they graduate from mandatory school at a later age compared to NF1-free persons, with males being more delayed than females. Contrary to our hypothesis, we found no indication that medical and psychiatric diseases moderated the effect of NF1 on educational achievement.

The results of our nationwide study align with prior findings, e.g., a German sample that showed that about $30 \%$ $(N=68)$ of the participants with NF1 attained nine years of education or less [34]. Semi-structured interviews of 60 Australian adults with NF1 revealed that $33 \%$ of the sample reached an educational level below high school and 25\% had a university degree [19]. We demonstrated higher or similar proportions of short and long education of 41 and $21 \%$, respectively, in our large nationwide study in a country with free (tax-financed) education at all levels. Importantly, these proportions were much higher for short and lower for long education when compared to persons not affected by NF1. Several potential mechanisms may play a role for educational outcome including cognitive functioning, certain academic skills such as reading or spelling, learning disabilities and medical and psychiatric disease burden. Supporting cognitive functioning as a potential 


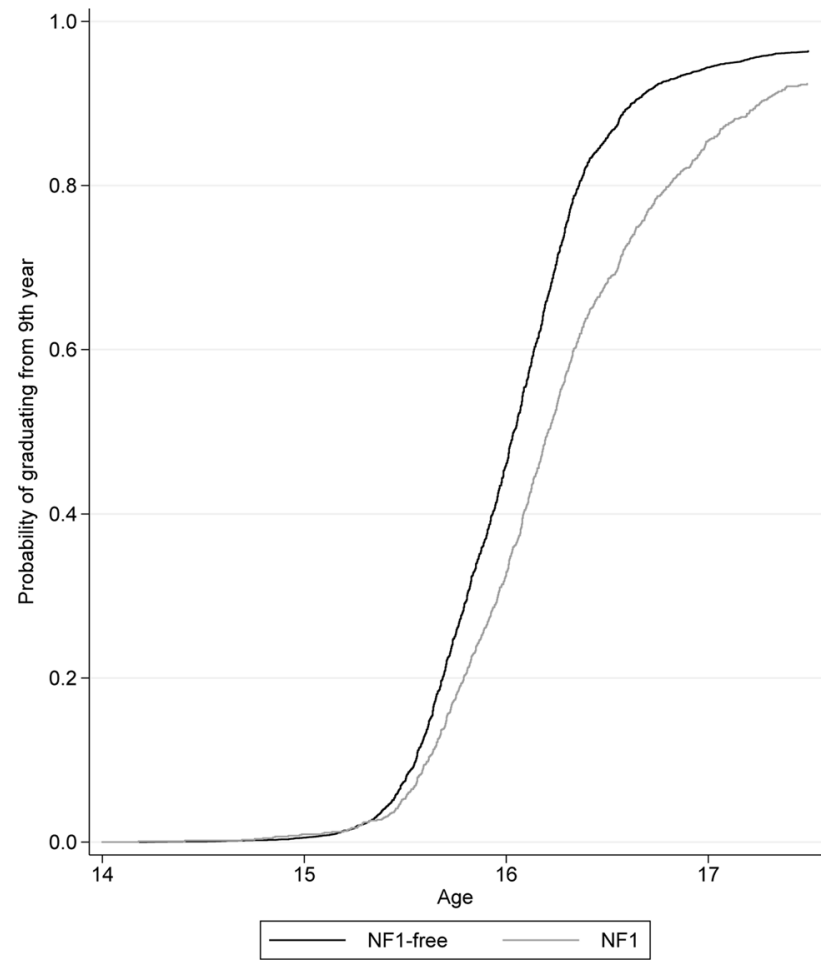

Fig. 4 a, b Cumulative probability of graduating from year 9 at different ages by NF1 status for persons born 1965-2000 (a) and

Table 4 Effect on age at graduating from $9^{\text {th }}$ year of mandatory school in 932 persons with NF1 born between 1965 and 2000 given as percentiles for graduating with $95 \%$ CI, compared with 7962 NF1-free persons

\begin{tabular}{|c|c|c|c|c|c|c|}
\hline & \multicolumn{2}{|c|}{$\begin{array}{l}\text { Model } 1^{\mathrm{a}} \\
(n=9856)\end{array}$} & \multicolumn{2}{|c|}{$\begin{array}{l}\text { Model 2 } \\
(n=9638)\end{array}$} & \multicolumn{2}{|c|}{$\begin{array}{l}\text { Model } 3 \\
(n=9638)\end{array}$} \\
\hline & Effect & $\mathrm{CI}$ & Effect & $\mathrm{CI}$ & Effect & $\mathrm{CI}$ \\
\hline \multicolumn{7}{|c|}{ 10th percentile } \\
\hline NF1-free & 1 & . & 1 & . & 1 & . \\
\hline NF1 & 1.05 & $1.02-1.08$ & 1.06 & $1.03-1.08$ & 1.05 & $1.02-1.08$ \\
\hline \multicolumn{7}{|c|}{ 25th percentile } \\
\hline NF1-free & 1 & . & 1 & . & 1 & . \\
\hline NF1 & 1.07 & $1.04-1.09$ & 1.07 & $1.04-1.09$ & 1.06 & $1.04-1.09$ \\
\hline \multicolumn{7}{|l|}{ Median } \\
\hline NF1-free & 1 & . & 1 & . & 1 & . \\
\hline NF1 & 1.09 & $1.07-1.11$ & 1.09 & $1.07-1.11$ & 1.08 & $1.05-1.10$ \\
\hline \multicolumn{7}{|c|}{ 75th percentile } \\
\hline NF1-free & 1 & . & 1 & . & 1 & . \\
\hline NF1 & 1.14 & $1.10-1.17$ & 1.12 & $1.09-1.15$ & 1.11 & $1.08-1.14$ \\
\hline \multicolumn{7}{|c|}{ 90th percentile } \\
\hline NF1-free & 1 & . & 1 & . & & \\
\hline NF1 & 1.26 & $1.19-1.33$ & 1.21 & $1.12-1.29$ & . & . \\
\hline
\end{tabular}

CI confidence interval

${ }^{\text {a }}$ Model 1 adjusted for birth year group and gender

${ }^{\mathrm{b}}$ Model 2 adjusted for birth year, gender and mother's education

${ }^{c}$ Model 3 adjusted for birth year, gender, mother's education, somatic and psychiatric diseases

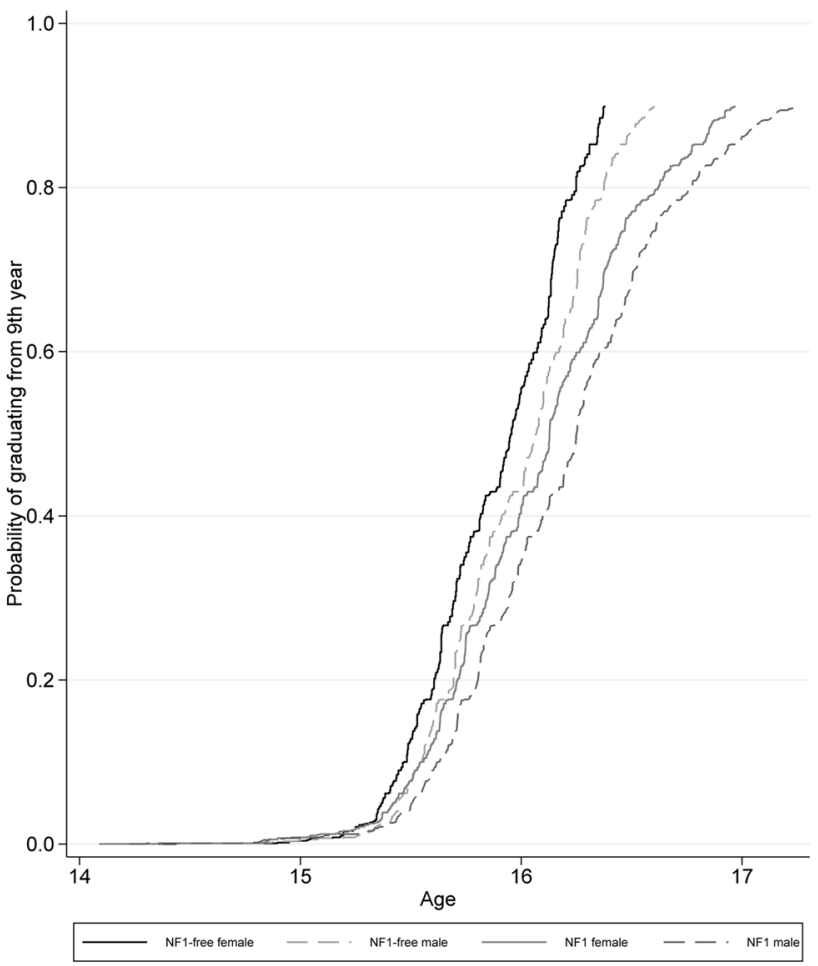

cumulative probability of graduating from year 9 by gender and NF1 status for persons born 1975-1979 (b), Denmark, 1981-2015

mechanism, it has been reported that the Intelligence Quotient (IQ) in the majority of children with NF1 lies within the normal range, but roughly 10 points lower [12]. However, intelligence alone may not play an important role in educational achievement. Additionally, children with NF1 have been shown to have lower performance in academic skills [21] such as reading [18] and higher prevalence of dyslexia and dyscalculia [15] compared to NF1-free children, resulting in lower academic achievements. Such impairments might strongly contribute to the lower levels of the highest attained education found in this nationwide study. Lehtonen et al. concluded in a systematic review based on 57 studies that children with NF1 do worse in most academic tasks compared to their siblings, comparison children, or standardized norms [11, 16, 21, 35, 36]. Learning disabilities are not only present during childhood and school time. Granström found in a questionnaire-based survey that two-thirds of a cross-sectional sample of 228 adults with NF1 reported current learning problems [34]. We did not find any effect modification on by medical or psychiatric diseases and infer that more subtle and possibly undiagnosed cognitive (e.g. ADHD [37]) and physical problems not resulting in hospitalizations may account for children who are able to finish lower secondary school but who are graduating at a later age. This is consistent to findings by Coudé et al. that $53 \%$ of persons with NF1 had 
repeated their school grade and $67 \%$ were found to be inattentive [38]. Further, Pride et al. showed that a comorbid diagnosis with ADHD affects some aspects of cognitive functioning and learning compared to children with only NF1 as well as unaffected children [39]. This calls for further research to investigate underlying reasons and how cognitive functioning may affect the educational attainment in persons with NF1.

Our study design does not allow us to disentangle the direct causes for delays in graduation from mandatory school or to identify if delay is primarily due to later start at school, repetitions of school years through school or a combination of these. However, the physical symptoms and their treatments, cognitive impairments, learning disabilities and lower academic performances probably all contributed to the significant delays of persons with NF1 in primary and lower secondary school.

Over time, the education level in the general population have increased, which was also observed in the current study. This might be the result of the high investments in the Danish pre-primary and primary education and further development of the education system [40]. However, we have to consider that reaching a university degree is not necessarily the educational aim for all persons. Not only cognitive and physical functioning, but also motivation, ambition, social skills and familial resources as well as peer group expectations contribute to the individual choice of a specific educational attainment. In our analyses, we accounted for calendar time and for socioeconomic family background by using mother's education.

Among those graduating early, differences between persons with and without NF1 are modest, but among the oldest persons with NF1 to graduate, $26 \%$ are older than their NF1free peers. This may suggest heterogeneity among persons with NF1 [7], where persons, who are less delayed in graduation may be different in terms of how NF1 affects them (e.g. in terms of disease severity and consequently in hospitalizations and other health service needs). However, further studies are needed to address these possible differences.

The strengths of the study include the nationwide population-based design with inclusion of all persons diagnosed with NF1 and no loss of follow-up using data collected independently of study hypotheses. The large sample size enabled sub-analyses using almost complete data on both covariates and outcomes, which strengthen the generalizability of the results. The access to nationwide health registries ensured information on medical and psychiatric diseases, used as proxies for severity of NF1. Further, we were able to take into account the mother's education, which is a well-known predictor for the educational level of children [41] thus minimizing confounding bias.

The present study also has limitations. Although identification of the persons with NF1 relied on diagnoses retrieved from the hospital register, medical records have not been examined to verify the diagnosis, and we thus cannot exclude misclassifications. Even though this was one of the largest cohort studies on educational attainment, some sub-analyses had low statistical power. Further, to evaluate the impact of missing information on mother's education, we applied multiple imputation, which did not affect the results. We chose to focus on the first major educational milestone, graduation from lower secondary school, as this is the point in time where each individual first chooses his or her educational trajectory. Further substantial delays during education beyond mandatory school among persons with NF1 may thus underestimate ultimate educational attainment delay.

\section{Conclusions}

This nationwide study demonstrates that persons with NF1 have educational delays starting as early as in basic school with the potential to persist during adulthood and that they are less likely to attain medium or higher educational level. These findings have implications for schools, health professionals and municipalities to provide adequate support for students with $\mathrm{NF} 1$ in order to ensure the highest possible educational level according to the potential and wishes of each person with NF1. Early interventions and specific therapeutic approaches targeting the specific disorders and disabilities that play a role for educational attainment should be implemented in order to improve social functioning and increase participation of persons with NF1 in society as well as work market affiliation.

Acknowledgements This work was supported by the U.S. Army Medical Research and Materiel Command, through the Neurofibromatosis Research Program under Award No. W81XWH-14-10054. Opinions, interpretations, conclusions and recommendations are those of the author and are not necessarily endorsed by the U.S. Army.

\section{Compliance with ethical standards}

Conflict of interest The authors declare that they have no conflict of interest.

Publisher's note: Springer Nature remains neutral with regard to jurisdictional claims in published maps and institutional affiliations.

\section{References}

1. Williams VC, Lucas J, Babcock MA, Gutmann DH, Korf B, Maria BL. Neurofibromatosis type 1 revisited. Pediatrics. 2009;123:124-33.

2. D Gareth R Evans, Catherine O'Hara, Anna Wilding, Sarah L Ingham, Elizabeth Howard, John Dawson, et al. Mortality in 
neurofibromatosis 1: in North West England: an assessment of actuarial survival in a region of the UK since 1989. Eur J Hum Genet. 2011;19:1187-91.

3. Tu Anh Duong, Emilie Sbidian, Laurence Valeyrie-Allanore, Cédric Vialette, Salah Ferkal, Smaïl Hadj-Rabia, et al. Mortality associated with neurofibromatosis 1: a cohort study of 1895 patients in 1980-2006 in France. Orphanet J Rare Dis. 2011;6:18.

4. Rasmussen Sa, Yang Q, Friedman JM. Mortality in neurofibromatosis 1: an analysis using U.S. death certificates. Am J Hum Genet. 2001;68:1110-8.

5. Ferner RE. Neurofibromatosis 1 and neurofibromatosis 2: a twenty first century perspective. Lancet Neurol. 2007;6:340-51.

6. Ozonoff S. Cognitive impairment in neurofibromatosis type 1. Am J Med Genet. 1999;89:45-52.

7. Boyd K, Korf B, Theos A. Neurofibromatosis type 1. J Am Acad Dermatol. 2009;61:1-16.

8. Friedman JM, Birch PH. Type 1 neurofibromatosis: a descriptive analysis of the disorder in 1,728 patients. Am J Med Genet. 1997;143:138-43.

9. Costa RM, Silva AJ. Molecular and cellular mechanisms underlying the cognitive deficits associated with neurofibromatosis 1 . J Child Neurol. 2002;17:646-51. 622-9

10. Garwood MM, Bernacki JM, Fine KM, Hainsworth KR, Davies WH, Klein-Tasman BP. Physical, cognitive, and psychosocial predictors of functional disability and health-related quality of life in adolescents with neurofibromatosis-1. Pain Res Treat. 2012;2012:975364.

11. Lianne C Krab, Femke K Aarsen, Arja de Goede-Bolder, Coriene E Catsman-Berrevoets, Willem F Arts, Henriette A Moll, et al. Impact of neurofibromatosis type 1 on school performance. J Child Neurol. 2008;23:1002-10.

12. Hyman SL, Shores A, North KN. The nature and frequency of cognitive deficits in children with neurofibromatosis type 1 . Neurology. 2005;65:1037-44.

13. Descheemaeker M, Plasschaert E, Frijns J, Legius E. Neuropsychological profile in adults with neurofibromatosis type 1 compared to a control group. J Intellect Disabil Res. 2012;1:1-13.

14. Kenborg L, Duun-Henriksen AK, Dalton SO, Bidstrup PE, Doser $\mathrm{K}$, Rugbjerg K, et al. Rates and Nature of Hospitalisations in Neurofibromatosis 1: A Danish population-based cohort study. 2019 (in preparation).

15. Orraca-Castillo M, Estévez-Pérez N, Reigosa-Crespo V. Neurocognitive profiles of learning disabled children with neurofibromatosis type 1. Front Hum Neurosci. 2014;8:386.

16. Cutting LE, Levine TM. Cognitive profile of children with neurofibromatosis and reading disabilities. Child Neuropsychol. 2010;16:417-32.

17. Gilboa Y, Rosenblum S, Fattal-Valevski A, Toledano-Alhadef H, Josman $\mathrm{N}$. Is there a relationship between executive functions and academic success in children with neurofibromatosis type 1 ? Neuropsychol Rehabil. 2014;2011:1-18.

18. Watt SE, Shores A, North KN. An examination of lexical and sublexical reading skills in children with neurofibromatosis type 1 . Child Neuropsychol. 2008;14:401-18.

19. Hilda A Crawford, Belinda Barton, Meredith J Wilson, Yemima Berman, Valerie J McKelvey-Martin, Patrick J Morrison, et al. The impact of neurofibromatosis type 1 on the health and wellbeing of Australian adults. J Genet Couns. 2015;24:931-44.

20. Barton B, North K. Social skills of children with neurofibromatosis type 1. Dev Med Child Neurol. 2004;46:553-63.

21. Coudé FX, Mignot C, Lyonnet S, Munnich A. Academic impairment is the most frequent complication of neurofibromatosis type-1 (NF1) in children. Behav Genet. 2006;36:660-4.
22. Granström S, Friedrich RE, Langenbruch AK, Augustin M, Mautner VF. Influence of learning disabilities on the tumour predisposition syndrome NF1-Survey from adult patients' perspective. Anticancer Res. 2014;34:3675-81.

23. Pedersen CB, Gøtzsche H, Møller JO, Mortensen PB. The Danish Civil Registration System. a cohort of eight million persons. Dan Med Bull. 2006;53:441-9.

24. Lynge E, Sandegaard JL, Rebolj M. The Danish National Patient Register. Scand J Public Health. 2011;39:30-3.

25. Jensen VM, Rasmussen AW. Danish education registers. Scand J Public Health. 2011;39:91-4.

26. EaGE M for C. Overview of the Danish education system. 2016. http://eng.uvm.dk/general-overview/overview-of-the-danisheducation-system. Accessed 09 February 2019.

27. UNESCO. International standard classification of education ISCED 1997. p.48. (UNESCO Institute for Statistics: Montreal, Quebec, Canada) 2006.

28. Mors O, Perto GP, Mortensen PB. The Danish Psychiatric Central Research Register. Scand J Public Health. 2011;39: 54-7.

29. Williams RL, Box PO, Carolina N. A note on robust variance estimation for cluster-correlated data. Biometrics. 2000; 56:645-6.

30. Cohen JS, Levy HP, Sloan J, Dariotis J, Biesecker BB. Depression among adults with neurofibromatosis type 1: prevalence and impact on quality of life. Clin Genet. 2015;88:425-30.

31. Augusto Pasini, Adriana Lo-Castro, Loredana Di Carlo, Mariabernarda Pitzianti, Martina Siracusano, Caterina Rosa, et al. Detecting anxiety symptoms in children and youths with neurofibromatosis type I. Am J Med Genet B Neuropsychiatr Genet. 2012;159B:869-73.

32. StataCorp. Stata Statistical Software: Release 14. College Station, TX: StataCorp LP. 2015.

33. SAS Institute Inc. SAS 9.1.3. SAS Institute Inc. 2005.

34. Granstrom S, Friedrich RE, Langenbruch AK, Augustin M, Mautner V-F. Influence of learning disabilities on the tumour predisposition syndrome NF1--survey from adult patients' perspective. Anticancer Res. 2014;34:3675-81.

35. Hyman S, Shores A, North KN. Learning disabilities in children with neurofibromatosis type 1: subtypes, cognitive profile, and attention-deficit-hyperactivity disorder. Dev Med Child Neurol. 2006;48:973-7.

36. Descheemaeker MJ, Ghesquière P, Symons H, Fryns JP, Legius E. Behavioural, academic and neuropsychological profile of normally gifted Neurofibromatosis type 1 children. J Intellect Disabil Res. 2005;49:33-46.

37. Ginsberg Y, Beusterien KM, Amos K, Jousselin C, Asherson P. The unmet needs of all adults with ADHD are not the same: a focus on Europe. Expert Rev Neurother. 2014;14: 799-812.

38. Coudé FX, Mignot C, Lyonnet S, Munnich A. Early grade repetition and inattention associated with Neurofibromatosis Type 1. J Atten Disord. 2007;11:101-5.

39. Pride NA, Payne JM, North KN. The Impact of ADHD on the cognitive and academic functioning of children with NF1. Dev Neuropsychol. 2012;37:590-600.

40. European Commission. Education and training monitor 2017 Denmark. Education and training. Vol. 2. Luxemburg: Publications Office of the European Union. 2017.

41. Koch S, Kejs AMT, Engholm G, Johansen C, Schmiegelow K. Educational attainment among survivors of childhood cancer: a population-based cohort study in Denmark. $\mathrm{Br} \mathrm{J}$ Cancer. 2004;91:923-8. 